\title{
Improved Understanding of the Sensitivity of Linear Tetrapolar Impedance Measurement (TPIM) and 8-Electrode Focused Impedance Method (FIM) in a Volume Conductor
}

\author{
Sayed Parvez Ahmed ${ }^{1,2, *}$, M Abdul Kadir ${ }^{1}$, Golam Dastegir Al-Quaderi³ \\ Rubina Rahman ${ }^{2}$ and K Siddique-e Rabbani ${ }^{1}$ \\ ${ }^{1}$ Department of Biomedical Physics \& Technology, University of Dhaka, Dhaka-1000, Bangladesh \\ ${ }^{2}$ Department of Physics, Jahangirnagar University, Savar, Dhaka, Bangladesh \\ ${ }^{3}$ Department of Physics, University of Dhaka, Dhaka-1000, Bangladesh \\ Email: parphyfield@yahoo.com, kadir@du.ac.bd, dastegir@gmail.com, rabbani@du.ac.bd \\ ${ }^{*}$ Corresponding author
}

\begin{abstract}
Tetrapolar Impedance Measurement (TPIM) is a 4-electrode impedance measurement system appropriate for a volume conductor in which current is driven through a pair of electrodes while potential developed across another pair of electrodes is measured to provide a value of transfer impedance. The 8-electrode Focused Impedance Method (FIM-8) consists of two concentric and orthogonal linear TPIM systems with their transfer impedances added for the purpose of localizing the central zone. Detailed 3D sensitivity studies are necessary for proper application of the techniques in specific biomedical applications and most reported work present point sensitivity distributions. The present work mainly focuses on planar average sensitivity in planes parallel to the electrode plane and its variation with depth due to different combinations of electrode separations - both for current drive pair and the potential measuring pair. This was obtained through finite element simulation using COMSOL Multiphysics software for a $40 \times 40 \times 40 \mathrm{~cm}^{3}$ volume. The results give useful information that can be used to design electrode configurations and measurement modalities for various applications.
\end{abstract}

Keywords: Electrical Impedance, Localized impedance, TPIM, FIM, Finite Element Method, Planar sensitivity.

\section{Introduction}

Electrical Impedance techniques have potential in many areas of biomedical applications. Among the simplest techniques, Tetrapolar Impedance Measurement (TPIM) and Focused Impedance Method (FIM) are the two most popular ones. A typical linear arrangement of electrodes in a traditional Tetrapolar Impedance Measurement (TPIM) method is shown in Figure 1. Here current is passed through a pair

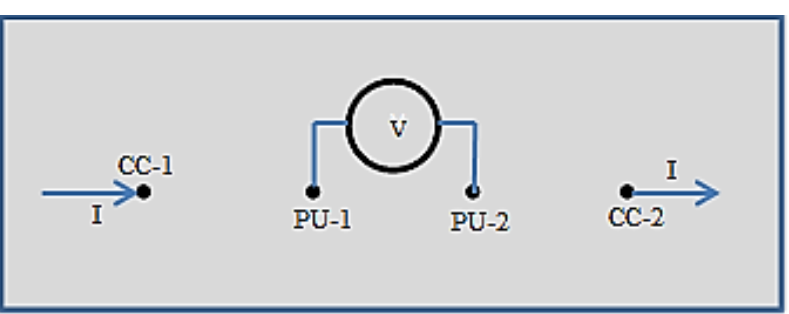

Fig-1: Electrode configuration in traditional Linear Tetrapolar Impedance Measurement (TPIM) system. of current drive electrodes (CC-1 and CC-2) and potential is measured across another pair (PU-1 and PU-2). A value of transfer impedance (Brown et al, 2000, Martinsen and Grimnes, 2009) is obtained dividing the potential $V$ by the driven current $I$.

Focused Impedance Method (FIM) was conceived by the Bio-medical physics group in the department of Physics of the University of Dhaka (Rabbani et al. 1999, Rabbani and Karal, 2008). It has three versions having 8, 6 and 4 electrodes respectively. The 8-electrode FIM essentially consists of two concentric orthogonal linear TPIM Systems where the individual transfer impedances are averaged to 
give enhanced sensitivity in the central region. The electrode configuration is shown in Figure 2. Here CC-1 and CC-2 are the current drive electrodes and PU-1 and PU-2 are the potential measuring electrodes for the first TPIM giving $V_{l}$ as the measured potential. Again, $\mathrm{CC}-3$ and $\mathrm{CC}-4$ are the current drive electrodes for the second TPIM for which PU-3 and PU-4 are the potential measuring electrodes giving $V_{2}$ as the measured potential. Typically both driven currents have the same value so that the transfer impedance in FIM is given by $\left(V_{I}+V_{2}\right) / I$. This has enhanced contribution from the central region for which it is said to be focused. This can be used effectively to localize organs or objects of interest in volume conductors like the human body.

An understanding of the 3D sensitivity in a volume

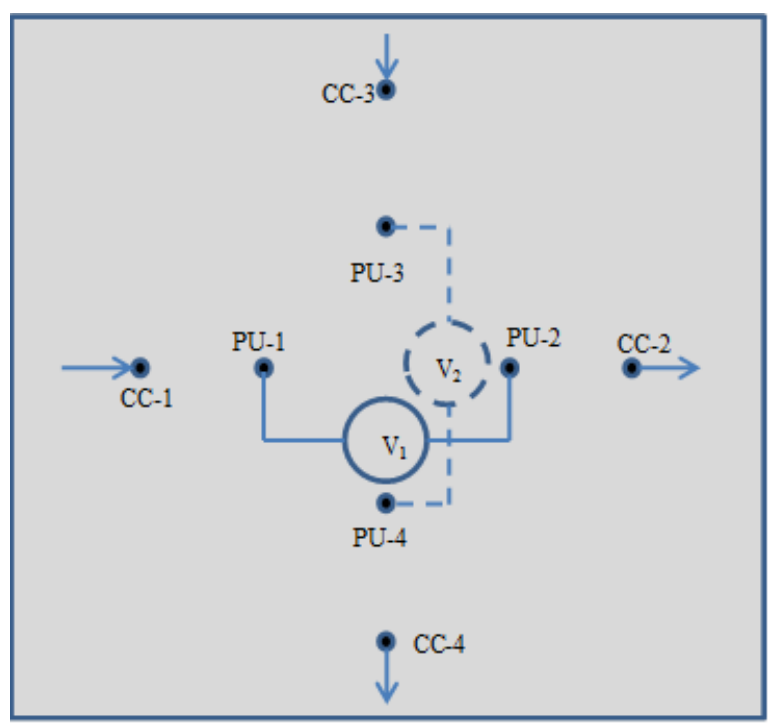

Fig-2: Electrode configuration in 8-electrode FIM. It is essentially two linear TPIM systems placed orthogonally. conductor of the above two measurement configurations would be useful in a choice for particular applications. This paper particularly concentrates on the variation of planar average sensitivity at different depths for different separations of current drive and potential electrodes.

The sensitivity (S) of a particular point within a volume conductor in TPIM is given by the dot product of two current density vectors divided by the current squared (Martinsen and Grimnes 2009),

$$
S=\frac{\vec{J}_{1} \cdot \vec{J}_{2}}{I^{2}}
$$

Where $\vec{J}_{1}$ is the current density at the specified point due to a constant current $I$ driven through the pair of $\mathrm{CC}$ electrodes and $\vec{J}_{2}$ is the current density at the same point with the same constant current driven through the pair of PU electrodes.

For FIM, the transfer impedance was defined as the summation of the two orthogonal transfer impedances. Therefore, the sensitivity at any point for FIM was also defined as the sum of the sensitivities at that point due to the two orthogonal TPIM measurements (Islam et al, 2010).

A typical sensitivity pattern for TPIM at a depth very close to the electrodes is shown in Figure 3. It can be seen that it has a large negative sensitivity between the $\mathrm{CC}$ and PU electrodes on each side. This means if an

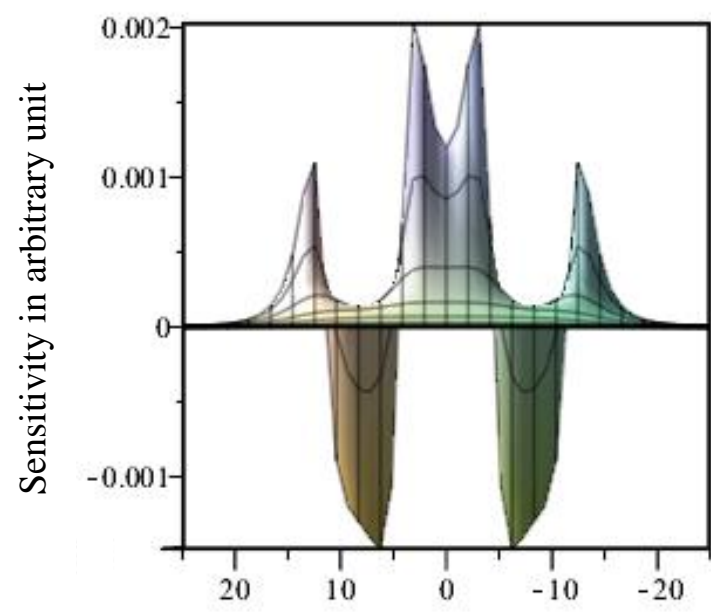

Distance along $\mathrm{X}$-axis in arbitrary unit

Fig-3: Numerical sensitivity of TPIM. Coordinates for CC electrodes were $(-12,0)$ and $(12,0)$ while that for PU electrodes were $(-4,0)$ and $(4,0)$. The negative sensitivity is seen at the regions between the $\mathrm{CC}$ and PU electrodes. 
object with a higher impedivity than that of the background is placed between the PU electrodes at the centre, the measured transfer Impedance will be greater than that measured for the background only. On the other hand if the same object is placed between the CC and PU electrodes on either of the two sides, the measured transfer Impedance will be smaller than that measured for the background only. Besides being asymmetric, the width of the sensitivity zone in $2 \mathrm{D}$ is expected to spread away from the line joining the electrodes in TPIM.

In order to limit the sensitivity zone at the centre, Focused Impedance Method (FIM) was proposed and successfully implemented in 2D (Rabbani et al 1999). Since then a number of papers reported the sensitivities of FIM in 2D and 3D (Abir et al, 2014; Islam et al, 2010; Iquebal and Rabbani, 2010; Saha et al, 2013) and its possible application in physiological studies and diagnosis in the areas of gastric emptying, lungs ventilation, breast tumour characterization, abdominal fat thickness measurement, etc., (Ahmed et al, 2014; Al-Quaderi et al, 2014; Amin et al, 2014; Ferdous et al, 2013; Howlader et al, 2010; Rabbani and Kadir, 2011; Surovy et al, 2012).

However, for a proper application to investigate particular geometries and situations in the human body it is necessary to have a thorough understanding of the methods that are being used.

In many applications the overall sensitivity of a plane at a certain depth is of concern rather than a point sensitivity distribution which most reports deal with. Besides, in both TPIM and FIM, the separation of CC and PU electrodes are of particular concern. The sensitivity patterns are expected to vary significantly with these parameters. This paper investigates the variations in planar average sensitivity with depth for different separations of CC and PU electrodes both for TPIM and FIM using finite element simulation which helps in improved understanding while analyzing a particular experimental result.

\section{Methods and Materials}

For the present work a finite element based software package known as 'COMSOL Multiphysics' was used (ac/dc module, version 4.3a). A cube shaped phantom $\left(40 \times 40 \times 40 \mathrm{~cm}^{3}\right)$ was taken to be the bulk conductor with conductivity and relative permittivity as $1 \mathrm{~S} / \mathrm{m}$ and 10 respectively. Copper electrodes of $1 \mathrm{~cm}$ diameter were placed on one surface of this cubical volume. An alternating current with a frequency of $5 \mathrm{KHz}$ and constant amplitude of $1 \mathrm{~A}$ was applied to the current driving electrodes.

From COMSOL Multiphysics, the pixel level values of sensitivity were exported to a worksheet using which the planar average sensitivity within the bulk volume considered (40 cm cube) were calculated for different planes parallel to the electrode plane, at different depths. Planar average sensitivity values were calculated for 11 planes starting from zero depth, i.e., at the electrode plane, to a depth of $10 \mathrm{~cm}$ below electrode surface, at $1 \mathrm{~cm}$ intervals. The planar average sensitivity values were then plotted against depth for different Electrode separations. In the present work, two sets of readings were taken, in the first, the PU electrode separation was kept fixed at $6 \mathrm{~cm}$ for both TPIM and FIM and the CC electrode separations were taken as $12 \mathrm{~cm}, 14 \mathrm{~cm}, 16 \mathrm{~cm}$ and $18 \mathrm{~cm}$ respectively. In the other set, the CC electrode separation was kept fixed at $18 \mathrm{~cm}$ while the PU electrode separations were taken at $6 \mathrm{~cm}, 8 \mathrm{~cm}$, $10 \mathrm{~cm}$ and $12 \mathrm{~cm}$ respectively

Simulation was also performed to study the variation at small depths in a greater detail, from zero to $0.25 \mathrm{~cm}$ at $0.05 \mathrm{~cm}$ intervals. The results are given in the next section. 


\section{Results and Observations}

Typical 2D sensitivity distributions for TPIM and FIM, as obtained using this simulation are shown in Figure 4. For these plots the separation of the CC electrodes was $18 \mathrm{~cm}$ and that of the PU electrodes was $6 \mathrm{~cm}$, and the figures show the point sensitivity distribution in a plane at a depth of $3 \mathrm{~cm}$ from the electrode plane.
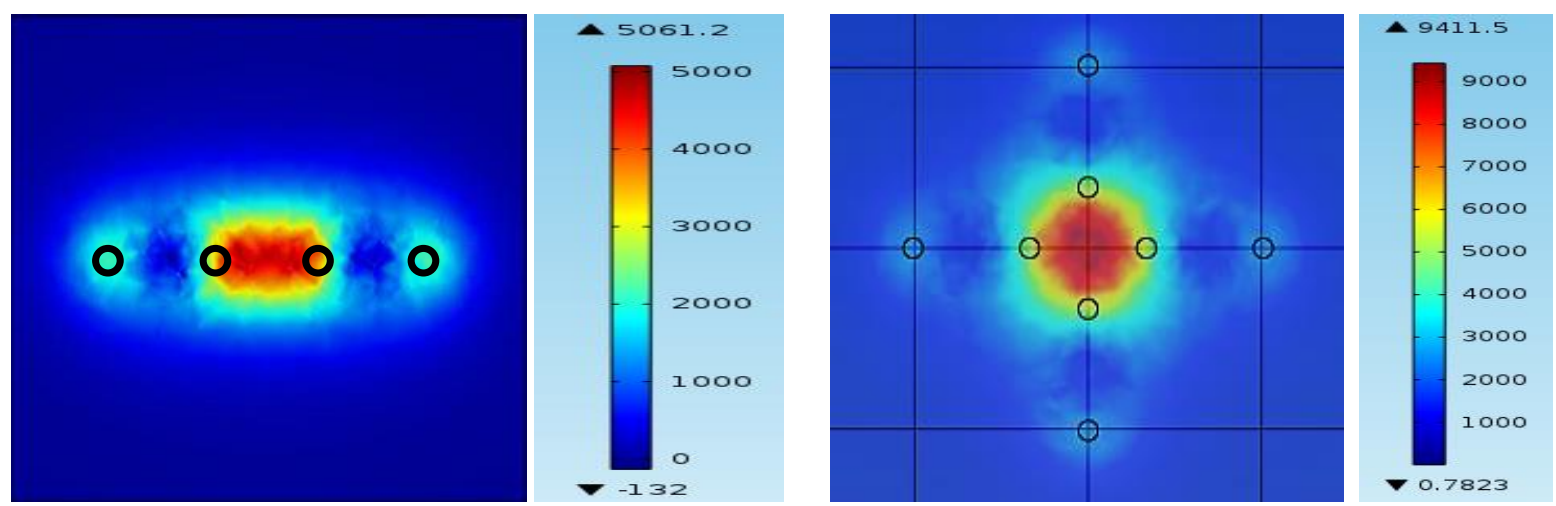

Fig-4: Point sensitivity distribution for TPIM (left) and FIM (right) at 3cm depth below electrode surface as simulated in Comsol Multiphysics software. For both, the CC electrode separation was $18 \mathrm{~cm}$ and PU electrode separation was $6 \mathrm{~cm}$. The electrode positions are indicated by the superimposed circles. The FIM gives a symmetric localization of sensitivity.

Negative sensitivity can be seen at regions between the CC and PU electrodes for TPIM, but not for FIM. This can be appreciated from the minimum values shown numerically at the base of the colour bar, which are -132 and +0.7823 respectively. In FIM, positive sensitivity due to one configuration of TPIM cancels out the negative sensitivity due to the other orthogonal configuration. However, it will be shown later that negative sensitivity exists to some extent at shallower depths. The symmetry of FIM is also to be noted. Both of these factors make FIM a useful modality for measurement for practical applications.

For the planar average sensitivity, the results obtained for TPIM and FIM for a constant PU electrode separation of $6 \mathrm{~cm}$ and for different $\mathrm{CC}$ electrode separations (indicated in the figures) are shown in Figures 5 and 6 respectively. It can be seen that for a CC separation of $12 \mathrm{~cm}$, the peak value occurs at the same value for both TPIM and FIM. This is expected because of the close relationship between TPIM and FIM. In this case the separation between the CC and PU electrodes (CC-PU separation) on each side is $3 \mathrm{~cm}[=(12-6) / 2]$ while the peak value occurs at about $1 \mathrm{~cm}$, at about $1 / 3^{\text {rd }}$ the CC-PU separation. This was also observed by others (Brown et al, 2000, Islam et al 2010). The positions of the peaks at the other CC separations also support this observation.

As mentioned above, for the FIM, the sensitivity values for the two orthogonal TPIM configurations were summed, therefore, the numerical values for FIM are higher than the corresponding ones for TPIM, being almost double. However, an interesting variation may be observed in the magnitude of negative sensitivity at shallow depths. For TPIM with fixed PU of $6 \mathrm{~cm}$, the negative value is high for $12 \mathrm{~cm} \mathrm{CC} \mathrm{electrode} \mathrm{separation.} \mathrm{It} \mathrm{then} \mathrm{vanishes} \mathrm{and} \mathrm{starts} \mathrm{with} \mathrm{a} \mathrm{high} \mathrm{positive} \mathrm{sensitivity} \mathrm{for} 14 \mathrm{~cm} \mathrm{CC}$ separation, and again decreases and goes towards a negative value for $18 \mathrm{~cm} \mathrm{CC}$ separation. For FIM, the behavior is similar but the negative value is much smaller, in comparison with respective positive values. 
Another point worth noting is that the variation of the values with depth goes down with increasing CC separation, giving a more uniform sensitivity over a range of depth, which may be useful in certain situations.

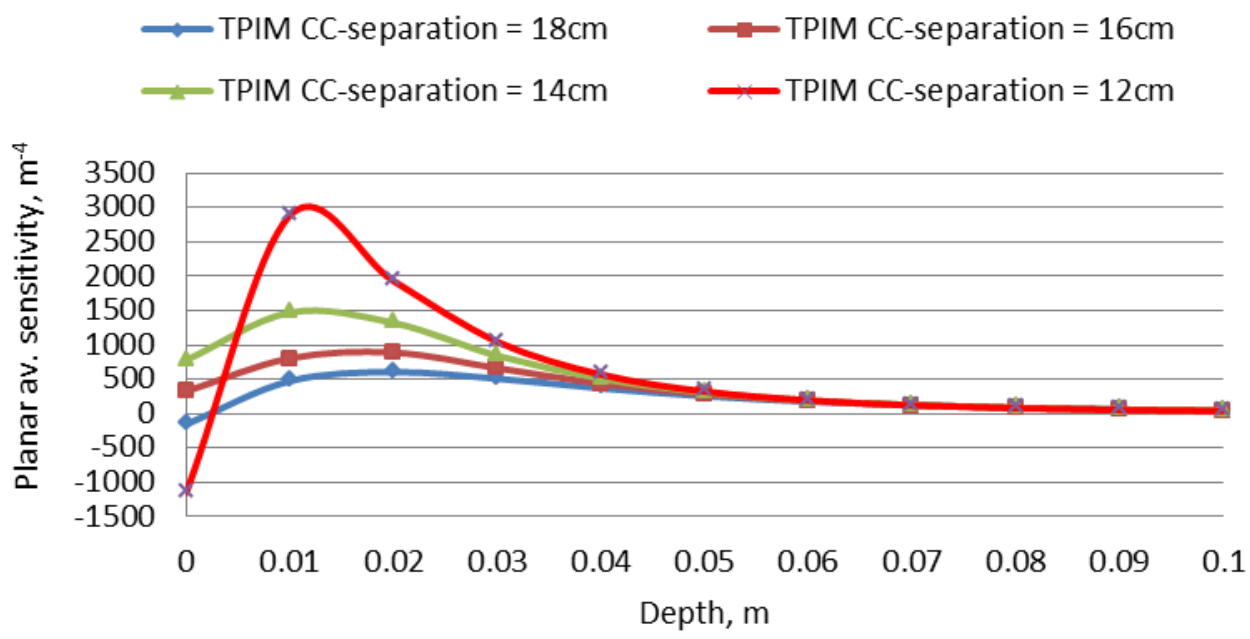

Fig 5: Planar average sensitivity for TPIM, plotted against depth for different CC electrode separations with a constant PU electrode separation of $6 \mathrm{~cm}$

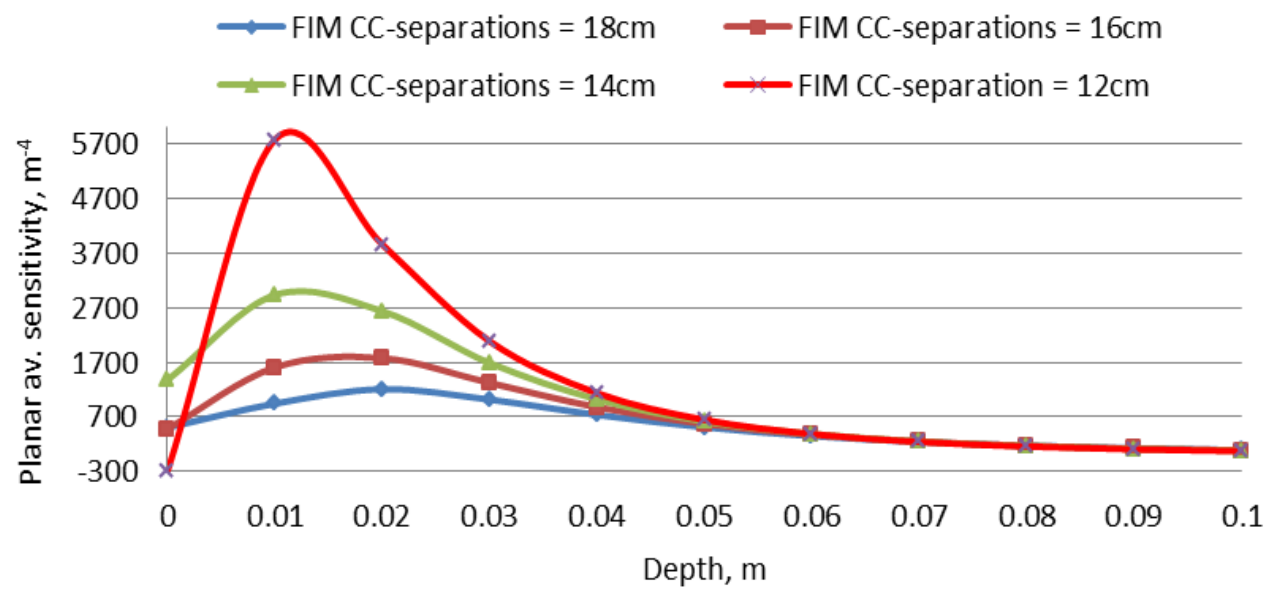

Fig 6: Planar average sensitivity for 8-electrode FIM, plotted against depth for different $\mathrm{CC}$ electrode separations with a constant PU electrode separation of $6 \mathrm{~cm}$.

Figure 7 and 8 shows the planar average sensitivities for both TPIM and FIM respectively for different PU electrode separations with a fixed CC electrode separation of $18 \mathrm{~cm}$. This also shows the interesting aspects with respect to negative sensitivity at shallow depths. The sensitivity goes towards negativity from a PU separation of $6 \mathrm{~cm}$ to about $10 \mathrm{~cm}$ after which it becomes positive at $12 \mathrm{~cm}$. In this case, the magnitude of negative sensitivity is more in FIM than in TPIM, in contrast with that obtained in Figures 5 and 6 respectively. 
Figures $9-12$ shows the details of the planar average sensitivities for similar conditions as for Figures $5-8$ respectively but for shallow depths only, less than $0.25 \mathrm{~cm}$. The behaviours appear anomalous, without any specific trend.
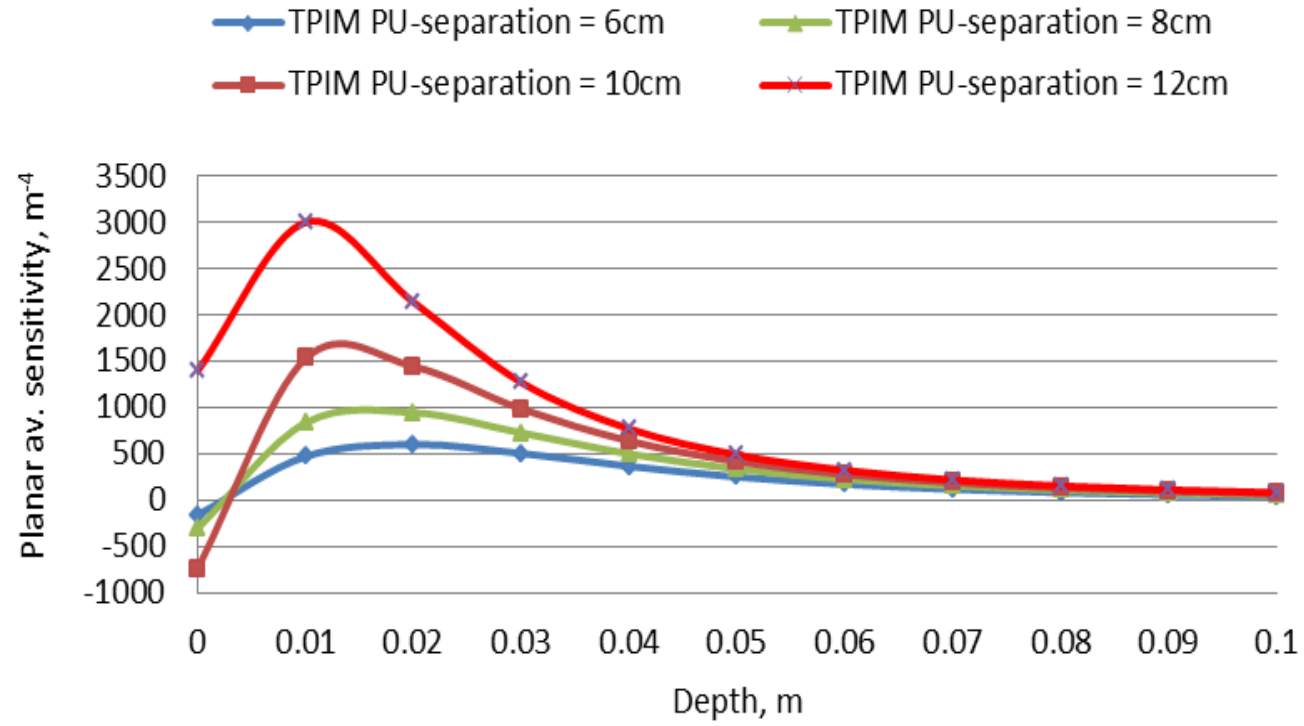

Fig 7: Planar average sensitivity with depth for TPIM for different PU separations. The $\mathrm{CC}$ electrode separation was fixed at $18 \mathrm{~cm}$.

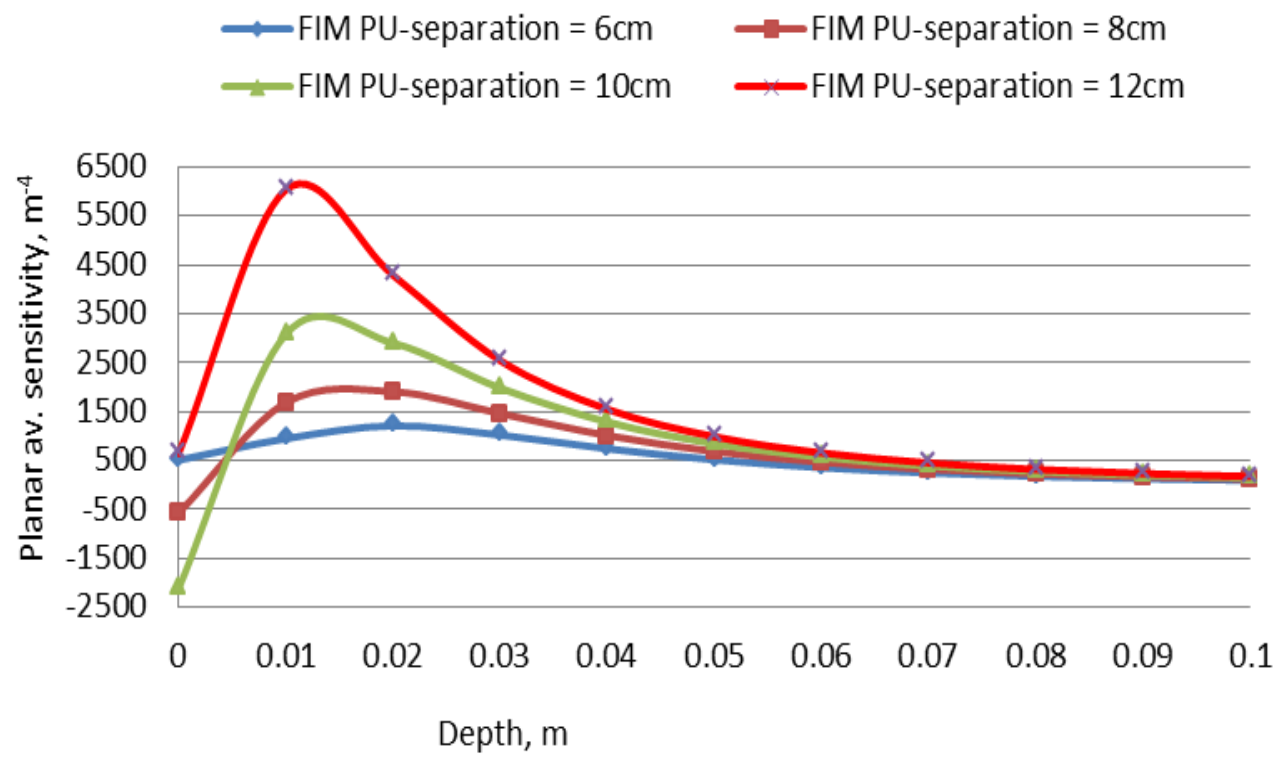

Fig 8: Planar average sensitivity with depth for 8-electrode FIM for different PU separations. The CC electrode separation was fixed at $18 \mathrm{~cm}$. 


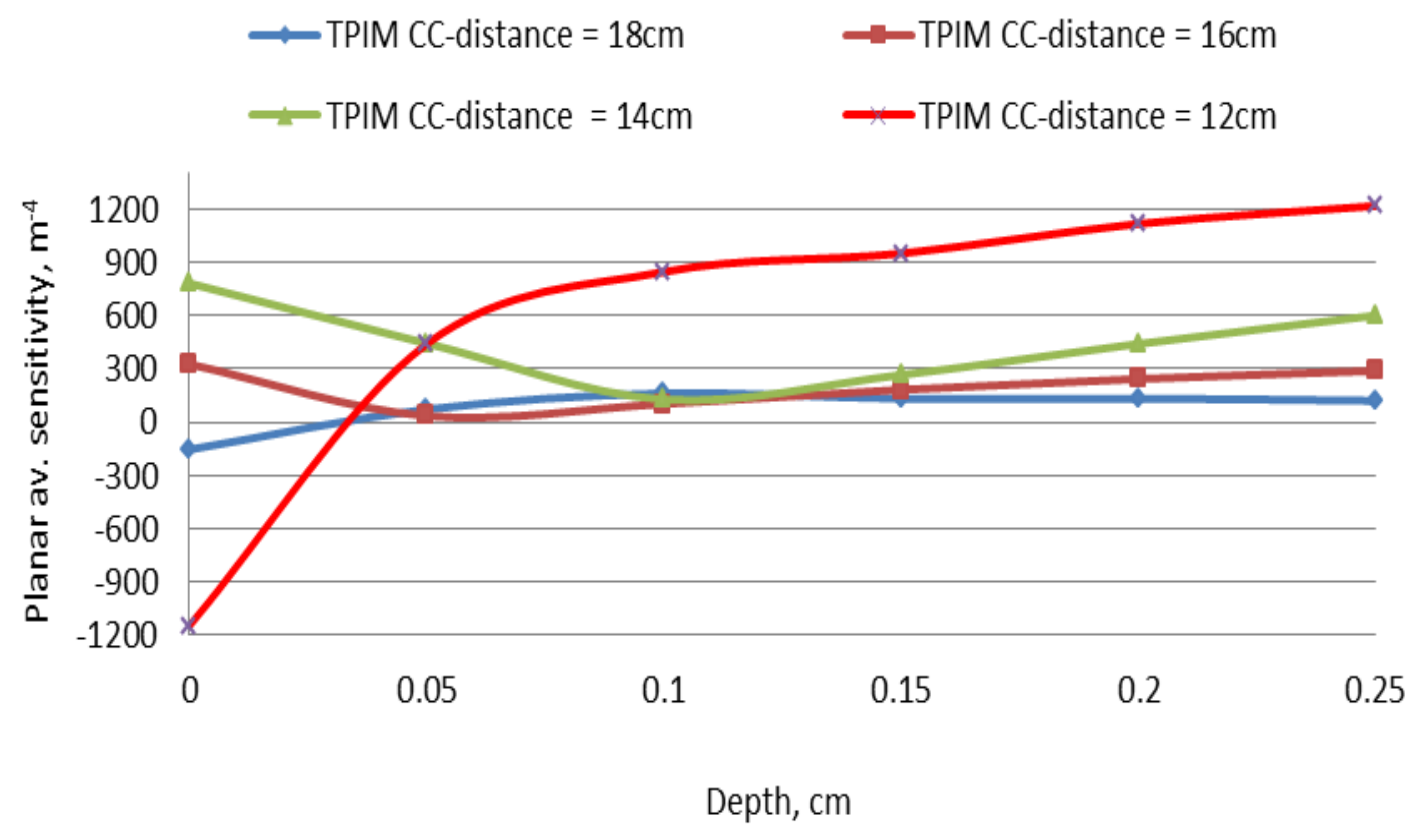

Fig 9: Details of planar average sensitivity with depth for TPIM at shallow depths, less than $0.25 \mathrm{~cm}$, for a fixed PU electrode separation of $6 \mathrm{~cm}$. It looks anomalous, because of several factors, including the electrode geometry.

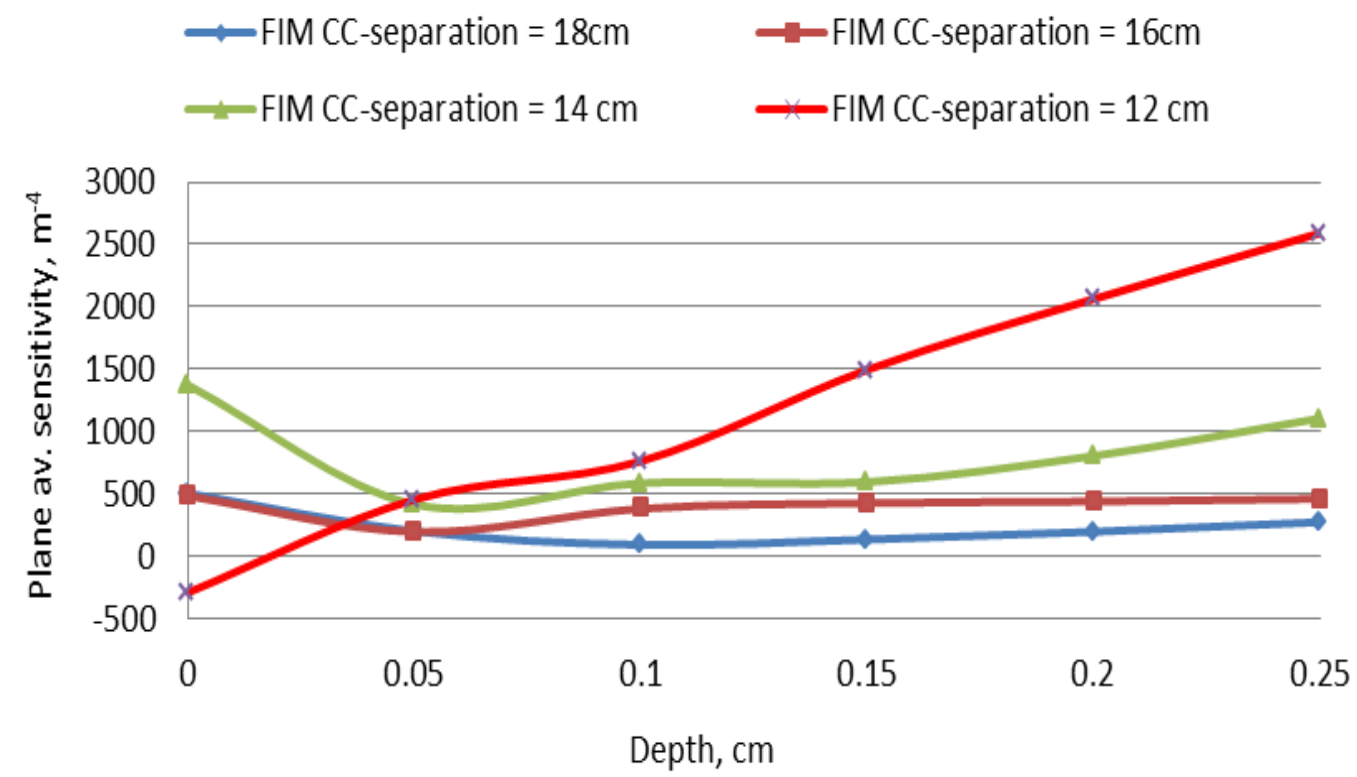

Fig 10: Details of planar average sensitivity with depth for FIM at shallow depths, less than $0.25 \mathrm{~cm}$, for a fixed PU electrode separation of $6 \mathrm{~cm}$. 


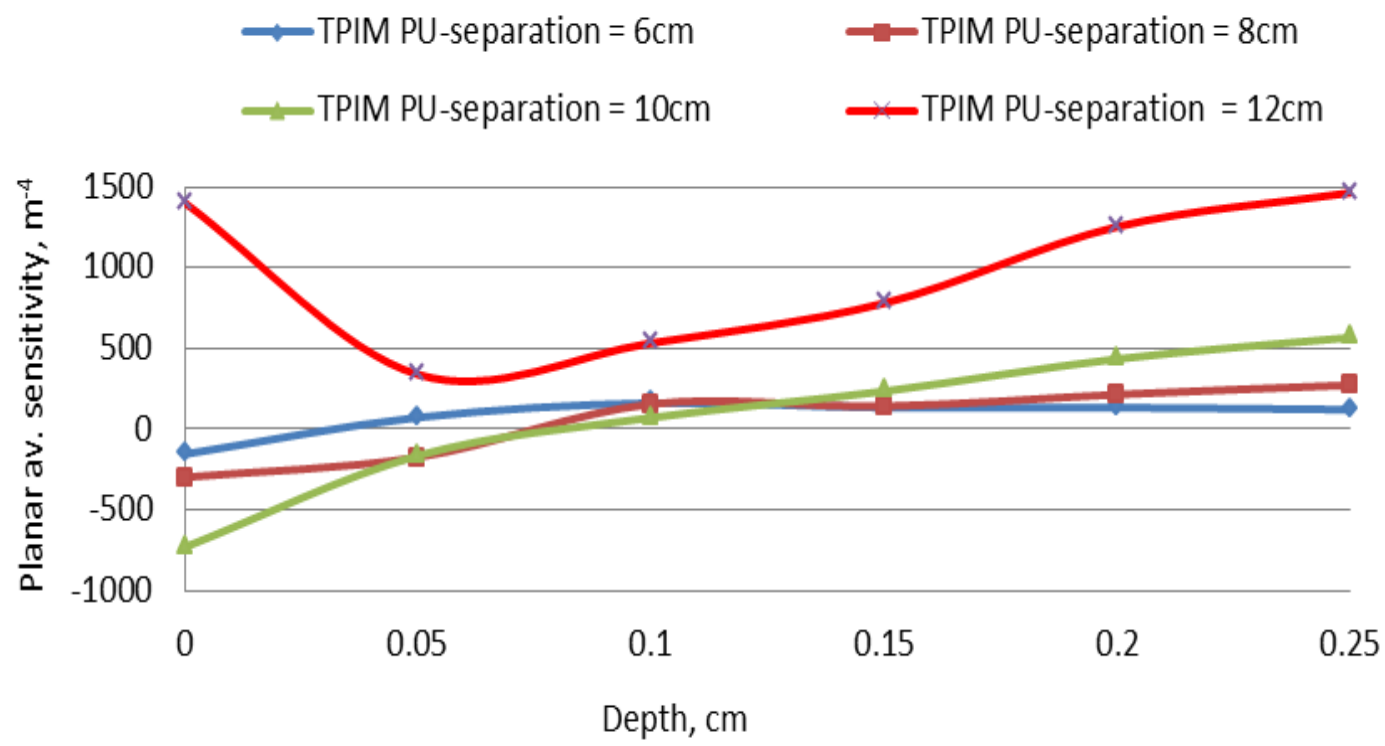

Fig 11: Details of planar average sensitivity with depth for TPIM at shallow depths, less than $0.25 \mathrm{~cm}$, for a fixed CC electrode separation of $18 \mathrm{~cm}$.

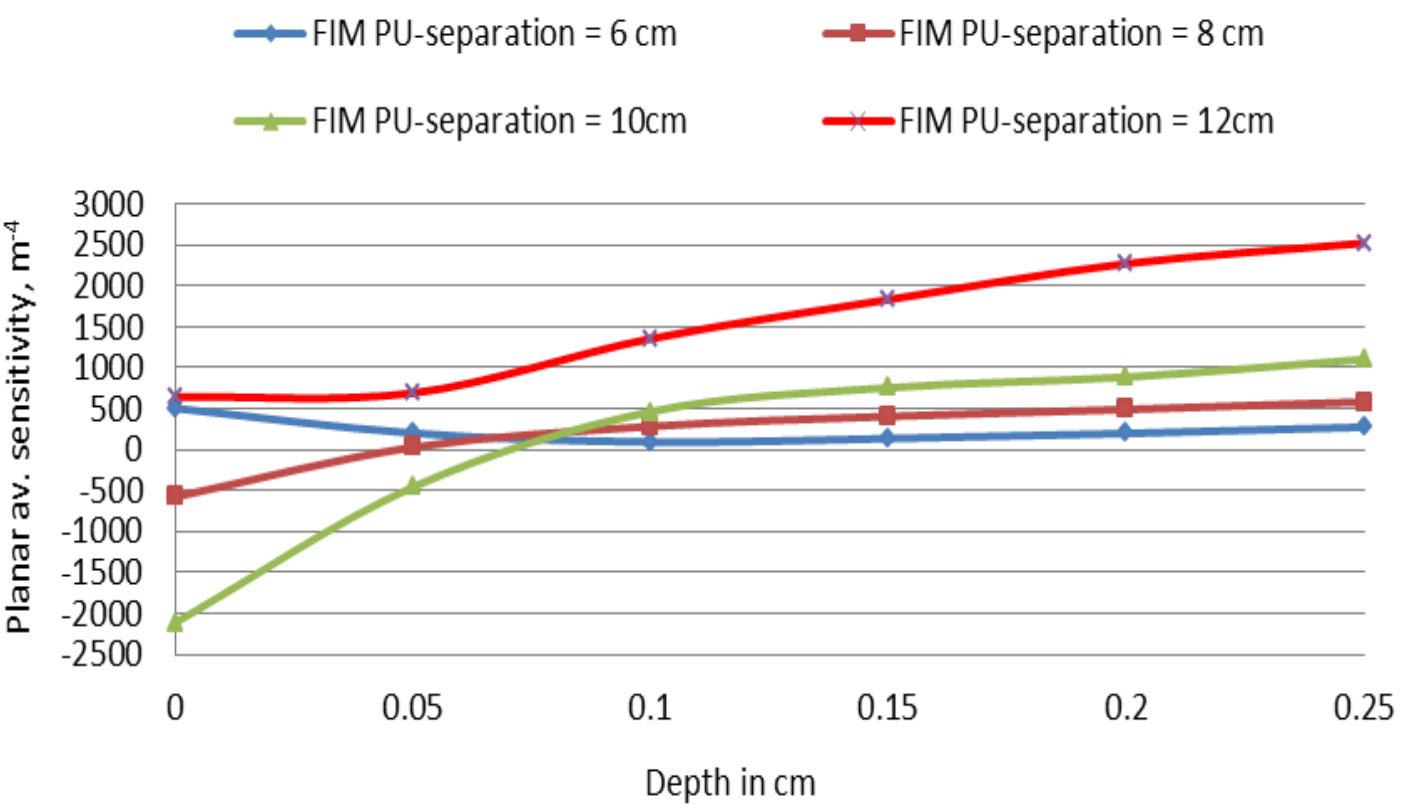

Fig 12: Details of planar average sensitivity with depth for FIM at shallow depths, less than $0.25 \mathrm{~cm}$, for a fixed CC electrode separation of $18 \mathrm{~cm}$. 


\section{Discussion}

In view of the potential applications of electrical impedance methods in Biomedical applications such systems should be understood and studied in detail. The present study looked into the effect of changing the current drive and potential electrode separations on TPIM and 8-electrode FIM systems, particularly for the planar average sensitivity at different depths for planes parallel to the electrode plane. Figures 5 to 8 show that for fixed CC electrode separation, as the separation between CC and PU electrodes decreases, the change in sensitivity with depth is more pronounced, with a peak value at one third of the CC-PU separation. This may be used intelligently to design TPIM and FIM systems to target a thin object at a particular depth. On the other hand if one desires to obtain an overall uniform sensitivity with depth, for objects that are extended in the $3^{\text {rd }}$ dimension, a large CC-PU separation may be chosen.

Again, Figures 9 to 12 suggest that at very shallow depths, less than $0.25 \mathrm{~cm}$ in these examples, the variation of planar average sensitivity with depth is anomalous, which may be due to the close proximity of electrodes as the electrode geometry may have a significant effect. Therefore, it is better to avoid targeting objects at such shallow depths with large electrodes, which in this case had diameters of $1 \mathrm{~cm}$.

Again, the point sensitivity distributions shown in Figure 4 support the primary conceptualization of FIM; FIM is indeed a better measurement to look for when studying a localized region or a localized object.

Acknowledgement: International Science Program (ISP) of Uppsala University, Sweden for financial support.

\section{References:}

Abir AR and Rabbani KS, 2014. Sensitivity study for a 4-electrode focused impedance method (FIM) using finite element method analysis. Bangladesh Journal of Medical Physics, 7: 1-7.

Ahmed SP, Kadir MA, Rahman R, Al-Quaderi GD and Rabbani KS, 2014. Determination of Organ Volume Using Focused Impedance Method (FIM): A Simulation Approach. Bangladesh Journal of Medical Physics, 7: 24-33.

Al-Quaderi GD, Ahmed SP and Rabbani KS, 2014. Determination of the thickness of a resistive material layer in a finite volume conductor using focused impedance method (FIM) - a simulation study. Bangladesh Journal of Medical Physics, 7:8-23.

Amin AA, Parvin S, Kadir MA, Tahmid T, Alam SK and Rabbani KS, 2014. Classification of Breast Tumour using Electrical Impedance and Machine Learning Techniques. Physiol Meas, 35: 965-974. doi:10.1088/0967-3334/35/6/965

Brown BH, Wilson AJ and Bertemes-Filho P, 2000. Bipolar and tetrapolar transfer impedance measurements from a volume conductor Electron. Electronics Letters, 36:2060-2061

Ferdous H, Baig TN and Rabbani KS, 2013. Thorax mapping for localized lung impedance change using focused impedance measurement (FIM): A pilot study. Journal of Electrical Bioimpedance, 4: 57-61.

Geselowitz DB, 1971. An application of electrocardiographic lead theory to impedance plethysmography. IEEE Trans Biomed Eng, 18: 38-41.

Haowlader S, Baig TN and Rabbani KS, 2010. Abdominal fat thickness measurement using Focused Impedance Method (FIM) - phantom study. J Phys: Conf Ser 224 (2010) 012061 (IOP Publishing). doi:10.1088/1742-6596/224/1/012061 
Iquebal AHM and Rabbani KS, 2010. 3D sensitivity of 6-electrode Focused Impedance Method (FIM). J Phys: Conf Ser 224 (2010) 012156 (IOP Publishing). doi:10.1088/1742-6596/224/1/012156

Islam N, Rabbani KS and Wilson AJ, 2010. The sensitivity of focused electrical impedance measurements. Physiological Measurement, 31:97-109, doi:10.1088/0967-3334/31/8/S08

Martinsen ØG and Grimnes S, 2009. The concept of transfer impedance in bioimpedance measurements. 4th European Conference of the International Federation for Medical and Biological Engineering, IFMBE Proceedings, 22:1078-1079.

Rabbani KS and Kadir MA, 2011. Possible applications of Focused Impedance Method (FIM) in biomedical and other areas of study. Bangladesh Journal of Medical Physics, 4: 67-74.

Rabbani KS and Karal MAS, 2008. A new four-electrode Focused Impedance Measurement (FIM) system for physiological study. Annals of Biomedical Engg (Springer), 36:1072-1077.

Rabbani KS, Sarker M, Akond MHR and Akter T, 1999. Focused Impedance measurement (FIM) - A new technique with improved zone localization Ann New York Acad Sci, 873:408-420.

Saha SK, Al-Quaderi GD and Rabbani KS, 2013. 3D Sensitivity of 8-Electrode FIM through Experimental Study in a Phantom. Bangladesh Journal of Medical Physics, 6:55-65.

Surovy NJ, Billah M, Haowlader S, Al-Quaderi GD and Rabbani KS, 2012. Determination of abdominal fat thickness using dual electrode separation in Focused Impedance Method (FIM). Physiol Meas, 33:707718. 